\title{
Epigenetic markers for molecular detection of prostate cancer
}

\author{
Vera L. Costa ${ }^{\mathrm{a}, 1}$, Rui Henrique ${ }^{\mathrm{b}, \mathrm{c}, 1}$ and Carmen Jerónimo ${ }^{\mathrm{a}, \mathrm{c}, \mathrm{d}, *}$ \\ ${ }^{a}$ Department of Genetics, Portuguese Oncology Institute - Porto, Rua Dr. Antonio Bernardino de Almeida, \\ 4200-072 Porto, Portugal \\ ${ }^{\mathrm{b}}$ Department of Pathology, Portuguese Oncology Institute - Porto, Rua Dr. Antonio Bernardino de Almeida, \\ 4200-072 Porto, Portugal \\ ${ }^{\mathrm{c}}$ Department of Pathology and Molecular Immunology, Institute of Biomedical Sciences Abel Salazar, University of \\ Porto, Largo Prof. Abel Salazar 2, 4099-003 Porto, Portugal \\ ${ }^{\mathrm{d}}$ Fernando Pessoa University School of Health Sciences, Rua Carlos da Maia, 296, 4200-150, Porto, Portugal
}

\begin{abstract}
Prostate cancer is a highly prevalent malignancy, which is clinically silent but curable while organ-confined. Because available screening methods show poor sensitivity and specificity, the development of new molecular markers is warranted. Epigenetic alterations, mainly promoter hypermethylation of cancer-related genes, are common events in prostate cancer and might be used as cancer biomarkers. Moreover, the development of quantitative, high-throughput techniques to assess promoter methylation enabled the simultaneous screening of multiple clinical samples. From the numerous cancer-related genes hypermethylated in prostate cancer only a few proved to be strong candidates to become routine biomarkers. This small set of genes includes GSTP1, APC, RAR 32 , Cyclin D2, MDR1, and PTGS2. Single and/or multigene analyses demonstrated the feasibility of detecting early prostate cancer, with high sensitivity and specificity, in body fluids (serum, plasma, urine, and ejaculates) and tissue samples. In addition, quantitative hypermethylation of several genes has been associated with clinicopathologic features of tumor aggressiveness, and also reported as independent prognostic factor for relapse. The identification of age-related methylation at specific loci and the differential frequency of methylation among ethnical groups, also provided interesting data linking methylation and prostate cancer risk. Although large trials are needed to validate these findings, the clinical use of these markers might be envisaged for the near future.
\end{abstract}

Keywords: Prostate cancer, epigenetics, methylation, molecular markers, biomarkers

\section{Introduction}

Prostate cancer has emerged as a leading health concern in recent years, standing as the most frequent noncutaneous malignant disease in men and the second leading cause of cancer related mortality, with an estimated 232,090 new cases and 30,350 deaths for the

\footnotetext{
${ }^{1}$ These authors contributed equally to this review and should be considered joint first authors.

${ }^{*}$ Corresponding author: Dr. Carmen Jerónimo, Department of Genetics, Portuguese Oncology Institute - Porto, Rua Dr. Antonio Bernardino de Almeida, 4200-072 Porto, Portugal. Tel.: +351 225084000 (ext. 5610); Fax: +351 225084016; E-mail: cjeroni@ ufp.pt.
}

year 2005 in the US [47]. Notwithstanding the dismal prognosis of locally advanced or metastatic disease, prostate cancer is a curable illness provided it is detected at its earliest stages, while still organ-confined. As effective prophylactic measures are not available and early-stage disease is often asymptomatic, highly efficient screening techniques are warranted.

The widespread use of serum prostate-specific antigen (PSA) determination constitutes a milestone event in early detection of prostate cancer [12]. The routine use of this cancer biomarker, in addition to digital rectal examination, resulted in an increase of organconfined prostate cancer detection rate [8], and may account for the decreasing mortality rate related with this 
disease [47]. However, the role of serum PSA levels as a screening tool for prostate cancer meets with important limitations. Although raised PSA levels (i.e., $>4.0 \mathrm{ng} / \mathrm{ml}$ ) are undoubtedly associated with the presence of prostate cancer, benign conditions, such as prostatitis and benign prostatic hyperplasia (BPH), which is common in the elderly, are also a cause of elevated serum PSA. Consequently, there is a relatively high frequency of unnecessary prostate sextant biopsies, an invasive procedure which is expensive and uncomfortable for the patients [14]. Moreover, a significant proportion of men with PSA levels within the normal range harbor prostate cancer (up to 22\%) and a significant number of these show pathologic features of tumor aggressiveness $[13,68,69,85]$. Indeed, $21 \%$ of men with positive end of study biopsies enrolled in the Prostate Cancer Prevention Trial (PCPT) had serum PSA levels between 2.6 and $3.9 \mathrm{ng} / \mathrm{mL}$ and $15.4 \%$ of the tumors found in men with PSA levels $<2.5 \mathrm{ng} / \mathrm{mL}$ were high grade cancers [88]. Furthermore, there are also ethnic variations which influence the performance of the serum PSA test, as only $25-35 \%$ of Caucasians and Hispanics with a PSA in the $2-10 \mathrm{ng} / \mathrm{mL}$ range have positive biopsies for prostate cancer, whereas up to 70$80 \%$ of African-Americans in this same range have a positive biopsy [57]. Because the sensitivity and specificity of the serum PSA test is at best $75 \%$, this is a rather imperfect prostate cancer marker [70]. Although several PSA-derived indices have been developed (e.g., free PSA, complex PSA, PSA density, PSA velocity and doubling time), none has so far attained widespread acceptance. Finally, the PSA test is unable to accurately assess the threat that a prostate tumor poses to the patient's life. Usually, a patient diagnosed with localized prostate cancer will have his prostate surgically removed, although pathological findings indicate that approximately $15 \%$ to $30 \%$ of the tumors excised are clinically insignificant and, consequently, were unlikely to affect the patient lifespan or quality of life [61, 91].

Presently, ultrasound-guided needle sextant prostate biopsy is the standard method for prostate cancer diagnosis in its earliest stages [25]. This technique is safe, with a very low frequency of post-biopsy complications, and very accurate. Nonetheless, the tissue sample is rather small, which constitutes an important limitation for histopathological evaluation [25]. Indeed, interpretation of prostate core biopsies constitutes a difficult task for the pathologist because the diagnosis of cancer is often based on a limited number of malignant cells infiltrating among more numerous benign glands.
Moreover, the limited tissue sampling poses two important problems to the pathologist. First, the morphology of malignant prostate glands might be difficult to assess when the representation in the biopsy is scarce. Immunohistochemical techniques might help circumvent this problem, but none has been proven to definitely confirm or deny the diagnosis of prostatic malignancy [19]. Consequently, an important proportion (about 24\%) of men submitted to prostate biopsy because of increased serum PSA levels, suspicious ultrasonographic or clinical findings are found to harbor prostate cancer in repeat biopsies [55]. Second, in addition to cancer diagnosis, histopathological evaluation of prostate biopsies should also convey prognostic information that might guide clinical decision-making. However, from a purely statistical point of view, the sampled malignant glands might not be representative of the main tumor, resulting in frequent undergrading and understaging [86]. This problem is also augmented by the substantial interobserver variability of the most important prognostic parameter, i.e., the Gleason score [2]. Finally, even carcinomas with an identical morphology and stage may have quite different outcomes, thus limiting the usefulness of current prognostic indicators.

As it may be apparent from the previous statements, there is an urgent need for improving early detection, diagnosis and prognosis assessment in prostate cancer. Because routine histopathological evaluation has most probably reached its limits as a diagnostic and prognostic tool, the development of new, more sensitive and specific markers should be based on the identification of the actual mechanisms underlying prostate cancer, i.e., genomic alterations.

\section{Epigenetic alterations in prostate cancer}

Over the last few years, several genomic alterations have been consistently reported in prostate carcinoma (see [43] for a recent review). Many somatic alterations including mutations, gene deletions, gene amplifications, chromosomal rearrangements, and changes in DNA methylation are detectable in prostate cancer cells. These modifications probably accumulate over a period of several decades and the number of changes increases with disease progression. Although multiple alterations that appear to contribute to disease progression have been suggested, no single key change has been detected. Indeed, genetic alterations such as gene mutations are either infrequent (e.g., those that activate 
the Ras oncogene or inactivate the TP53 [44]) or occur late in disease progression (e.g., those that inactivate the tumor suppressor gene PTEN [10]). Hence, these alterations are not optimal candidates for cancer biomarkers in the clinic. Conversely, epigenetic events occur much more frequently and at earlier stages in prostate cancer, hypothetically providing more efficient tools for disease detection and management [51].

Two main categories of epigenetic alterations have been reported thus far in prostate cancer, i.e., histone modifications (including phosphorylation, methylation, and acetylation) and aberrant methylation (which includes both hypermethylation and hypomethylation) $[63,66,83]$. Most studies concerning the role of histone modifications in prostate cancer are based on the restoration of gene expression following treatment of cancer cells with histone deacetylases (HDAC) inhibitors. Although specific alterations have been reported for some genes (e.g., coxsackie and adenovirus receptor (CAR) and vitamin D receptor (VDR) $[3,78]$, it seems that alterations in the global levels of specific histone modifications are involved in prostate carcinogenesis and might also be independent outcome predictors [83].

DNA hypomethylation has also been seldom reported in prostate cancer. Interestingly, genomewide DNA hypomethylation was reported in $31 \%$ of prostate carcinomas and significantly correlated with aberrations in chromosome 8 and metastatic disease [82], thus linking hypomethylation with chromosome instability and disease progression. In addition, gene-specific methylation has been reported to affect several overexpressed genes in prostate cancer (e.g., PLAU, Urokinase, Heparanase, and Cytochrome P450 1B1 [74,76, $77,89]$ ) and might account for the activation of other proto-oncogenes in prostate carcinogenesis.

However, the most frequent and better characterized epigenetic alteration, not only in prostate carcinoma but also in other common human malignancies, is DNA hypermethylation [26], providing several promising cancer biomarkers [51]. A large number of cancer-related genes have been reported to be downregulated through promoter hypermethylation in prostate cancer (see reference [63] for a recent and comprehensive review) and several of these have shown promise for prostate cancer detection $[5,22,35,42,48,52,53])$. Besides the high frequency and early occurrence of this epigenetic alteration in prostate cancer, there are other important advantages in the use of DNA methylation as a biomarker for cancer detection. From a technical standpoint, DNA harboring methylation is more stable and easy to ma- nipulate than RNA, and simpler to extract than proteins. Moreover, hypermethylation is a positive signal that is less likely to be masked through contaminant normal DNA, making it particularly suited for detection in clinical samples, where sensitive detection is necessary owing to scarce tumor DNA or dilution by excess normal DNA. Finally, detection of hypermethylation in a large number of samples is now feasible using recent standardized high-throughput technologies, thus enabling its use in clinical practice.

\section{Methods for detection of DNA hypermethylation}

Several methodologies might be used for the identification of DNA methylated sequences. Some of them (e.g., bisulfite-sequencing) provide important information concerning the methylation profile and the degree of methylation heterogeneity of cells [16]. However, these methods are expensive, time-consuming, and not amenable to routine clinical use. Indeed, if a widespread use of DNA methylation markers for cancer detection is envisaged, assays characterized by high sensitivity, specificity, reproducibility, homogeneity and high-throughtput capabilities must be chosen. For this purpose, the assays based on the analysis of sodium bisulfite converted DNA [75] offer many advantages. The most widely used of these assays is methylation-specific PCR (MSP) [40] because it usually requires only minute quantities of template DNA, it is simple, safe and easy to perform, and it shows considerable sensitivity, which is a key requirement for the analysis of clinical samples.

In the original MSP method (conventional MSP, CMSP), PCR products are run in a gel and the results are reported as methylated or unmethylated at the target DNA sequence [40]. Consequently, this method does not allow the identification of partial levels of methylation, a feature which is extremely relevant both biologically and clinically. Thus, quantitative methods have been developed in recent years to overcome this limitation of CMSP, and the most important of these was the fluorescence-based real-time quantitative MSP (QMSP) assay [20]. In this assay, locus-specific primers, designed to amplify methylated CpGs, flank an oligonucleotide probe labeled with a 5' fluorescent reporter dye and a 3' quencher dye. During amplification, the Taq polymerase cleaves the reporter from the probe, owing to its 5'-3' exonuclease activity, thus releasing it from the quencher. Then, the monitoring of fluorescence emissions during the PCR process al- 
lows the quantitation of methylated alleles [37]. Because this quantity also depends on the input of template DNA, an internal reference should be used to normalize the assay. For this purpose, housekeeping gene (e.g., $M Y O D 1$ or $A C T B$ ) without $\mathrm{CpG}$ islands has been used by most researchers. However, recent findings indicate that Alu-based control reactions might be the best strategy to measure the input levels bisulfiteconverted DNA [92]. Several strategies might then be employed to express this quantity (e.g., a ratio calculated using the values obtained for the target gene and the reference gene in a given case) which represent the relative level of methylated gene in a given sample. The use of the probe improves the overall specificity of the assay owing to the more stringent amplification conditions. Moreover, the quantitative assay was shown to reliably detect promoter methylation in the presence of 10.000-fold excess of unmethylated alleles, i.e., a 10-fold increase in sensitivity over CMSP [20]. Consequently, high sensitivity and specificity are key features of QMSP. However, as incompletely methylated target sequences will not be amplified, the actual methylation levels might be greater than those determined. Notwithstanding this limitation of QMSP, its high-throughput characteristics are ideally suited for the analysis of large numbers of clinical samples.

\section{Gene promoter methylation and prostate cancer detection/diagnosis}

Among the relatively large number of cancer-related genes reported to be hypermethylated in prostate cancer, GSTP1 is unquestionably the most widely studied and well documented. This gene encodes a $\pi$-class Glutathione $S$-transferase (GST- $\pi$ ) enzyme involved in the detoxification of reactive chemical species by catalyzing their conjugation to reduced glutathione [36]. Thus, GSTP1 acts as a "caretaker" gene protecting prostate cells against genomic damage mediated by a variety of oxidants, including carcinogens [71]. Loss of GSTP1 function would predispose normal prostatic cells to endure DNA damage motivated by inflammation and/or dietary intake thus leading to carcinogenesis [72].

Since the first reports linking GSTP1 loss of expression with promoter methylation in prostate cancer $[58$, 59], many studies confirmed the high frequency of this epigenetic alteration in invasive tumor (36-94\%), as well as in prostatic intraepithelial neoplasia (PIN) (30$76 \%$ ) $[9,22,23,28,35,48,49,52,54,64,67,93-95]$. However, the tumor-specificity of GSTP1 hypermethylation is not absolute as morphologically normal prostate tissue and BPH might carry this alteration $[22,39,48,49]$. This problem is solved by the use of quantitative assays, most notably QMSP, because non-cancerous prostate tissues display only very low levels of GSTP1 promoter methylation [22,35,39,48,95,96].

Possibly, the most illustrative study concerning the power of GSTP1 hypermethylation as a prostate cancer marker in prostate tissues was performed by Harden and co-workers, which managed to compare the diagnostic accuracy of the test with the standard histopathological evaluation by an expert uropathologist [35]. In their report, the quantitative assessment of GSTP1 promoter methylation detected $11 \%$ more cases compared to histopathology, whereas a combination of both yielded a 15\% (79\% vs. 64\%) increase over histopathology alone [35]. Thus, a QMSP assay for GSTP1 might provide a valuable ancillary diagnostic tool for routine pathological assessment of prostate biopsies.

The better performance of the quantitative GSTP1 hypermethylation assay compared to standard histopathology might be explained by the detection of minute foci of tumor cells that would be insufficient to raise morphological suspicion of malignancy. Alternatively, the methylated GSTP1 alleles detected by the MSP assay might not only be derived from malignant cells but also from morphologically normal epithelial cells and/or stromal cells in the vicinity of the neoplastic glands. Thus, the epigenetic alterations detected in these morphologically normal cells would serve as surrogate markers for prostate cancer. This hypothesis is supported by a recent study which observed methylation at the GSTP1 and $R A R \beta 2$ promoters in non-neoplastic cells of prostate tumor microenvironment [34]. These findings could be due to a "fieldeffect" phenomenon and raise the possibility that epigenetic alterations in prostate tissue are associated with the exposure to carcinogens (either endogenous or exogenous) which seem to affect both epithelial and mesenchymal components. However, owing to the high prevalence of prostatic epithelial neoplasms and the rarity of mesenchymal tumors, it seems reasonable to assume that the acquisition of GSTP 1 and $R A R \beta 2$ promoter methylation in stromal cells is not sufficient to induce a malignant phenotype. Hence, the intrinsic biology of prostatic epithelial cells is likely to render them more susceptible to endure genomic alterations and subsequent neoplastic transformation.

Although the performance of the GSTP1 hypermethylation assay in tissue samples might perfect diagnostic accuracy in prostate biopsies, an important step 
forward in prostate cancer detection would be the development of a body fluid-based screening test. Such a test could increase the sensitivity and specificity of the serum PSA test, thus diminishing the number of unnecessary prostate biopsies. Circulating DNA in the plasma and serum of patients with urological malignancies has been documented [27]. Prostate cancer DNA may be present in the circulation as a result of intravascular cell death of prostate cancer cells or circulating phagocytic cells that have ingested prostate cancer cells [69]. Moreover, the anticipated shedding of neoplastic prostatic cells into the prostatic ducts raises the possibility of detecting cancer-related epigenetic alterations in urine sediments. Thus, voided urine and serum/plasma are obvious candidates for non- or minimally-invasive screening and have been tested accordingly $[11,28,29,31,50]$. However, two major concerns arose from these studies. First, the sensitivity of the test is significantly hampered (18.8-38.9\% for voided urine, and $13-72 \%$ for serum/plasma), although it might be augmented using prostatic massage prior to urine collection [29], collecting urine after prostate biopsy [31] or eventually by increasing the number of samples from each patient. Second, the test specificity is likely to be impaired when additional procedures are performed to increase the rate of detection [29,31]. The relatively high frequency of prostate cancer detection in ejaculates using a CMSP assay for GSTP1 hypermethylation $[28,30,87]$ might offer an additional source of test material. However, the nature of the sampling procedure, particularly in older men, meets with relevant ethical questions and practical limitations.

Several strategies might be anticipated to increase the sensitivity of prostate cancer detection in body fluids without compromising specificity. Thus, the combined use of an urine-based and a serum/plasma-based assay might increase the sensitivity of the test, as previously demonstrated [50]. Moreover, the use of a panel of genes surveyed for promoter methylation, in addition to GSTP1, might decisively increase both the sensitivity and the specificity of the test as verified in a recent report by Hoque and co-workers [42]. The gene panel, which included four genes (GSTP1, $p 16$, $A R F$, and $M G M T$ ), allowed those researchers to detect $87 \%$ of prostate carcinomas with $100 \%$ specificity, using a QMSP assay on urine sediments [42]. Similar gene panels, which variably include GSTP1, APC, $M D R 1, P T G S 2, R A R \beta 2$, and RASSF1A, have also been proposed to increase the sensitivity and specificity of prostate cancer detection in tissue samples [5,22,90, 95]. However, these panels should be viewed with some caution as the simultaneous use of more than 3 markers will only accomplish a minor increase in sensitivity at the cost of lowering the specificity of the test [95].

\section{Gene promoter methylation and prognosis prediction in prostate cancer}

Epidemiological data indicate that only a proportion of histologically diagnosed prostate carcinomas will progress to clinically significant and lethal disease whereas many patients die (from other causes) with an indolent form of prostate cancer [18]. Thus, tumors with overlapping morphological features may indeed be associated with a quite distinct disease outcome. As more effective screening tests are likely to increase the number of "clinically indolent" prostate cancers that would not benefit from aggressive forms of treatment, it is critical to develop markers that might identify the "clinically relevant" prostate carcinomas. Ideally, these markers would be tested in the clinical samples utilized for screening and/or diagnosis of prostate cancer and the results would guide patient management. Current prognostic indicators, including pre-treatment serum PSA level, clinical stage determined by digital rectal examination, and the tumor Gleason score on needle biopsy remain the mainstays used in clinical decision making [46]. However, the usefulness of these markers is limited as they do not allow for an accurate prognostic assessment in an individual basis. Consequently, and alongside with the reported up to $60 \%$ of clinically understaged prostate carcinomas [7], there is also an important proportion of prostate carcinomas treated by radical prostatectomy that might be better managed conservatively.

Several studies have documented statistical associations between clinical and pathological features of tumor aggressiveness (e.g., pathological tumor stage and Gleason score) and promoter methylation of single genes or gene panels including $A P C, C D H 1, E D N R B$, GSTP1, MDR1, MT1G, PTGS2, RAR 32 , RASSF1A, and $R U N X 3$ [5,21,22,38,39,52-54,60,84,95]. Interestingly, in a recent report a methylation (M) score, derived from the quantitative assessment of promoter methylation at the $G S T P 1, A P C$, and $M D R 1$ promoters, was able to discriminate organ-confined from locally advanced disease with $72.1 \%$ sensitivity and $67.8 \%$ specificity [22]. Although these results are still suboptimal for clinical application, they provide sufficient preliminary evidence to support further testing is larger trials. Curiously, the same M-score was found 
to be significantly higher in current smokers than in never smokers and to positively correlate with packyears smoked [23]. This is a rather unexpected finding as smoking has not been previously associated with prostate cancer risk [33].

In addition to assist in the therapeutic triage of prostate cancer patients, methylation markers might also provide valuable information concerning disease relapse prediction. Indeed, GSTP1 promoter methylation was found to be the most relevant predictor of early PSA recurrence in multivariate analysis in a recent study [4]. Moreover, $A P C$ and Cyclin D2 hypermethylation also seem to predict time to recurrence in a defined set of prostate carcinomas (i.e., tumors with Gleason score $3+4=7$ ) and were shown to be independent prognostic factors [79]. Unexpectedly, lower levels of GSTP1 promoter methylation were also reported to be associated with unfavorable outcome, eventually identifying a particular subset of prostate carcinomas that progress through alternative pathways [79].

It should be realized that the development of new prognostic markers for prostate cancer is difficult and complex owing to the heterogeneity of this neoplasm, which frequently manifests as multiple independent primary tumors within the same organ [73]. Histopathological assessment of radical prostatectomy specimens frequently reveals the existence of multiple, independent tumors, and this finding has been confirmed by genetic studies $[6,15,56,65,80]$. Thus, it is critical to determine whether independent prostatic tumor foci have comparable potential for invasion and metastasis. According to some reports, the characteristics of the "index" tumor in the prostatectomy specimen are those that more accurately predict the likelihood of recurrence and this is largely independent of the characteristics of the smaller, concurrent tumors [73]. Thus, the use of methylation markers for the pre-treatment assessment of prognosis in prostate cancer patients should obviously take in consideration the issue of prostate tumor heterogeneity.

\section{Gene promoter methylation and risk-assessment in prostate cancer}

Risk markers offer the benefit of providing an opportunity for early disease detection and diagnosis, thus augmenting the likelihood of curative treatment. Moreover, the use of these markers might allow the development of innovative prevention strategies, targeted to subjects at high risk, avoiding exposure of the whole population and thereby diminishing the potential problems related with the implementation of a screening test. In this vein, the refinement of the ability to identify individuals with high risk for prostate cancer would permit a less frequent screening in men at lower risk, consequently decreasing the false positive rate. The principal aim would be to augment the specificity of the screening and reduce the frequency of (unnecessary) prostate biopsies.

The search for risk-associated markers might then be guided by the known risk factors for prostate cancer, e.g., age, diet and genetic background [34]. Concerning age-related methylation, it is noteworthy that prostate cancer is primarily a disease of the elderly, with about $80 \%$ of the cases diagnosed after 65 years of age, and the average patient age at the time of diagnosis is 70 years. Moreover, the incidence is low prior to age 50 and increases exponentially until 80 years [47]. Previous studies documented that the acquisition of aberrant promoter methylation at several genes is an age-related phenomenon in normal human tissues [1]. Indeed, in colorectal mucosa, the methylation of a $\mathrm{CpG}$ island on the ESRI gene increases linearly with age [45]. Interestingly, methylation of ESRI has also been reported as both age-dependent and tumor differentiation-dependent in prostate cancer, thus representing a putative link between aging and prostate cancer [62]. Eventually, other cancer-related genes might become epigenetically inactivated in an age-dependent manner, thereby increasing the susceptibility of normal prostate cells to neoplastic transformation. Moreover, this hypothesis might substantiate the frequent, although at lower level, promoter methylation of specific genes reported in morphologically normal prostate glandular epithelium [39]. Hypothetically, individuals carrying these alterations in normal tissue might be at higher risk for developing prostate cancer and could benefit from more close surveillance.

Of all human cancers, prostate cancer shows the highest ethnic disparity in incidence and mortality [47]. When compared with Caucasians, African-Americans have a 1.7-fold higher incidence of cancer, twice the risk of developing advanced-stage prostate cancer, and a two- to threefold greater mortality rate [41]. Moreover, African-Americans are also more likely to have a family history of prostate cancer and are younger at the time of diagnosis. As socioeconomic, clinical, and pathological factors do not account for all these racial discrepancies [17], unknown race-related factors must be involved in prostate cancer susceptibility and progression. Conceivably, race-related patterns 


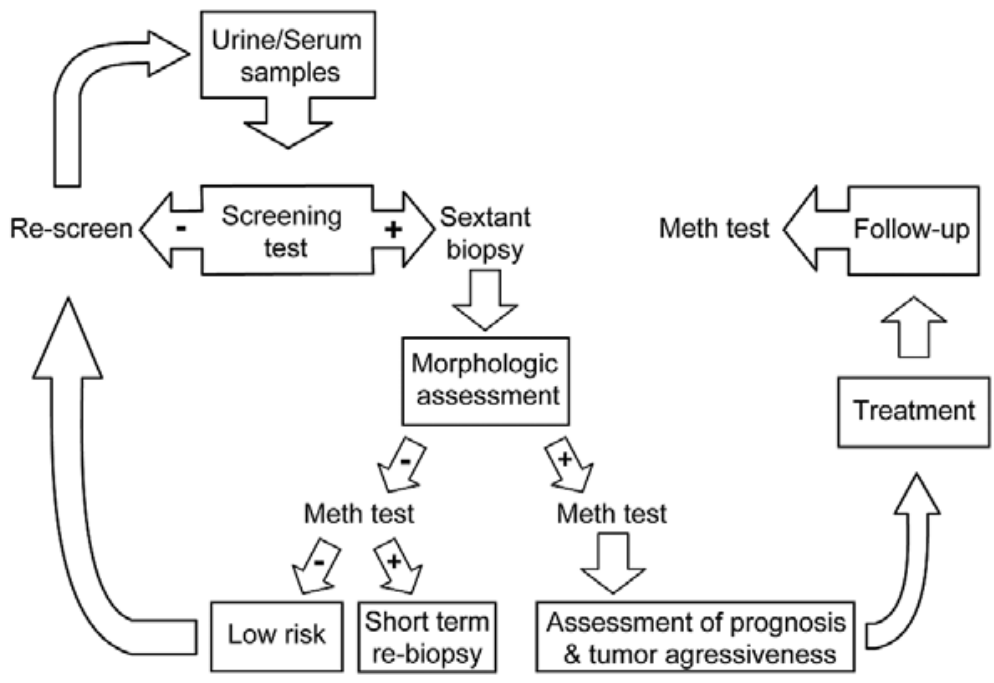

Fig. 1. Proposed algorithm for the management of prostate cancer suspects using epigenetic markers (Meth test, methylation markers-based test).

of gene methylation would be able to confer resistance or susceptibility to the development and/or progression of prostate cancer. Interestingly, racial differences in $C D 44$ gene promoter methylation have been reported, with a 1.7-fold higher frequency of methylation among African-Americans (43\%) relative to Caucasians (25\%) [93]. Differences in GSTP1 promoter methylation in prostate cancer among AfricanAmerican, Caucasian, and Asian populations have also been found. In a recent study, Enokida and co-workers reported that GSTP 1 methylation is a particularly good biomarker for prostate cancer in African-Americans whereas in Asian patients this epigenetic event correlates with pathological findings (higher $\mathrm{pT}$ categories and Gleason score) [23]. These findings suggest that GSTP1 hypermethylation is likely to have different performances as a prostate cancer biomarker in distinct ethnic groups.

Thus far, no substantial evidence has been provided linking DNA methylation with environmental factors (e.g., diet) or with hereditary susceptibility in prostate cancer.

\section{Application of epigenetic-based markers in prostate cancer detection and management: a proposal}

While clinicians and patients wait for the upcoming availability of commercial tests based on current knowledge about the prostate cancer epigenome, we are tempted to propose an algorithmic approach for the use of those tests in the clinic (Fig. 1). Ideally, this assay should include several epigenetic biomarkers (probably from 2 to 4 ) to maximize the sensitivity of the test and also to provide relevant information concerning the clinical significance of a prostate tumor. The development of multiplex assays is likely to be a useful and cost-effective methodology for that purpose. Following clinical evaluation, the test should be initially performed as a screening tool in healthy individuals at risk for prostate cancer. Thus, urine and/or serum would be the ideal clinical samples because its collection is not or minimally invasive. Individuals with a negative test would be re-tested within a given time period, probably 1 or 2 years, depending on the clinical evaluation. Conversely, a positive test should lead to sextant prostate biopsy. In addition to standard histopathological evaluation, testing for hypermethylation of the same set of genes in tissue samples (ideally from each side) may add relevant information for clinical management when adenocarcinoma is diagnosed. Conversely, if there is no morphological evidence of malignancy, a positive test should be followed by close surveillance and repeat biopsy within a short period of time. Eventually, this biopsy might be more extensive on the side from which the tissue sample tested positive was collected. Double negative cases (i.e., without morphological and molecular evidence of malignancy) would re-enter the screening path after clinical evaluation. Furthermore, patients diagnosed with prostate cancer might be monitored after treatment with a periodical blood and/or urine methylation test. This procedure might identify disease progression almost from its inception and triage 
patients for earlier and more adequate therapeutic procedures.

Please note that this is a purely speculative model and clinical evidence is still lacking to support many of the procedures. Moreover, the methylation test needs to be evaluated in large clinical trials to determine its usefulness and potential advantages over the currently available markers, such as serum PSA. However, this model may provide a rationale for the collection of the significant clinical and scientific evidence to back up the implementation of these new prostate cancer biomarkers in routine clinical practice.

\section{Conclusions}

Prostate cancer remains a significant challenge both to researchers and clinicians. Many questions concerning its biological characteristics and clinical significance await more definite answers. Clearly, current tools for prostate cancer screening, diagnosis, and management are limited, and innovative methodologies are needed. The hypermethylation of $\mathrm{CpG}$ islands represents a somatic, epigenetic event that almost uniformly arises during prostate carcinogenesis. Using modern detection assays, $\mathrm{CpG}$ island hypermethylation of multiple cancer-related genes has provided promising molecular markers for prostate cancer. Importantly, the detection of these alterations is readily feasible in routine clinical specimens such as urine, blood, and prostate biopsy samples, eventually providing critical information for risk-assessment, screening, diagnosis and treatment. Because epigenetic information is heritable but also has considerable plasticity, disease treatment and chemoprevention strategies might benefit from specific therapeutic methods that target epigenetic alterations. Hence, it is a reasonable expectation that epigenetic-based markers may decisively contribute for improved prostate cancer patient care in the future.

\section{Acknowledgements}

V.L.C. and C.J. are supported by grants from Fundação para a Ciência e a Tecnologia (SFRH/ BD/23374/2005 and Projecto de Investigação Plurianual do Centro de Investigação do IPO-Porto (03-05), respectively).

\section{References}

[1] N. Ahuja and J.P. Issa, Aging, methylation and cancer, Histology and Histopathology 15 (2000), 835-842.

[2] W.C. Allsbrook Jr., K.A. Mangold, M.H. Johnson, R.B. Lane, C.G. Lane and J.I. Epstein, Interobserver reproducibility of Gleason grading of prostatic carcinoma: general pathologist, Human Pathology 32 (2001), 81-88.

[3] C.M. Banwell, R. Singh, P.M. Stewart, M.R. Uskokovic and M.J. Campbell, Antiproliferative signalling by 1,25(OH)2D3 in prostate and breast cancer is suppressed by a mechanism involving histone deacetylation, Recent Results in Cancer Research 164 (2003), 83-98.

[4] P.J. Bastian, G.S. Palapattu, X. Lin, S. Yegnasubramanian, L.A. Mangold, B. Trock, M.A. Eisenberger, A.W. Partin and W.G. Nelson, Preoperative serum DNA GSTP 1 CpG island hypermethylation and the risk of early prostate-specific antigen recurrence following radical prostatectomy, Clinical Cancer Research 11 (2005), 4037-4043.

[5] P.J. Bastian, J. Ellinger, A. Wellmann, N. Wernert, L.C. Heukamp, S.C. Muller and A. von Ruecker, Diagnostic and prognostic information in prostate cancer with the help of a small set of hypermethylated gene loci, Clinical Cancer Research 11 (2005), 4097-4106.

[6] B. Beheshti, B. Vukovic, P. Marrano, J.A. Squire and P.C. Park, Resolution of genotypic heterogeneity in prostate tumors using polymerase chain reaction and comparative genomic hybridization on microdissected carcinoma and prostatic intraepithelial neoplasia foci, Cancer Genetics and Cytogenetics 137 (2002), 15-22.

[7] D.G. Bostwick, R.P. Myers and J.E. Oesterling, Staging of prostate cancer, Seminars in Surgical Oncology 10 (1994), 60-72.

[8] M.K. Brawer, Screening for prostate cancer, Seminars in Surgical Oncology 18 (2000), 29-36.

[9] J.D. Brooks, M. Weinstein, X. Lin, Y. Sun, S.S. Pin, G.S. Bova, J.I. Epstein, W.B. Isaacs and W.G. Nelson, CG island methylation changes near the GSTP1 gene in prostatic intraepithelial neoplasia, Cancer Epidemiology Biomarkers and Prevention 7 (1998), 531-536.

[10] P. Cairns, K. Okami, S. Halachmi, N. Halachmi, M. Esteller, J.G. Herman, J. Jen, W.B. Isaacs, G.S. Bova and D. Sidransky, Frequent inactivation of PTEN/MMAC1 in primary prostate cancer, Cancer Research 57 (1997), 4997-5000.

[11] P. Cairns, M. Esteller, J.G. Herman, M. Schoenberg, C. Jerónimo, M. Sanchez-Cespedes, N.H. Chow, M. Grasso, L. Wu, W.B. Westra and D. Sidransky, Molecular detection of prostate cancer in urine by GSTP1 hypermethylation, Clinical Cancer Research 7 (2001), 2727-2730.

[12] W.J. Catalona, D.S. Smith, T.L. Ratliff, K.M. Dodds, D.E. Coplen, J.J. Yuan, J.A. Petros and G.L. Andriole, Measurement of prostate-specific antigen in serum as a screening test for prostate cancer, New England Journal of Medicine 324 (1991), 1156-1161.

[13] W.J. Catalona, D.S. Smith and D.K. Ornstein, Prostate cancer detection in men with serum PSA concentrations of 2.6 to $4.0 \mathrm{ng} / \mathrm{mL}$ and benign prostate examination. Enhancement of specificity with free PSA measurements, Journal of the American Medical Association 277 (1997), 1452-1455.

[14] W.J. Catalona, P.C. Southwick, K.M. Slawin, A.W. Partin, M.K. Brawer, R.C. Flanigan, A. Patel, J.P. Richie, P.C. Walsh, P.T. Scardino, P.H. Lange, G.H. Gasior, K.G. Loveland and K.R. Bray, Comparison of percent free PSA, PSA density, 
and age-specific PSA cutoffs for prostate cancer detection and staging, Urology 56 (2000), 255-260.

[15] L. Cheng, S.Y. Song, T.G. Pretlow, F.W. Abdul-Karim, H.J. Kung, D.V. Dawson, W.S. Park, Y.W. Moon, M.L. Tsai, W.M. Linehan, M.R. Emmert-Buck, L.A. Liotta and Z. Zhuang, Evidence of independent origin of multiple tumors from patients with prostate cancer, Journal of the National Cancer Institute 90 (1998), 233-237.

[16] S.J. Clark, Studying mammalian DNA methylation: bisulfite modification, in: DNA Methylation. Approaches, Methods and Applications, M. Esteller, ed., CRC Press, Boca Raton, 2004, pp. 53-63.

[17] M.P. Cotter, R.W Gern, G.Y. Ho, R.Y. Chang and R.D. Burk, Role of family history and ethnicity on the mode and age of prostate cancer presentation, Prostate 50 (2002), 216-221.

[18] E.D. Crawford, Epidemiology of prostate cancer, Urology 62 (2003), 3-12.

[19] A.M. DeMarzo, W.G. Nelson, W.B. Isaacs and J.I. Epstein, Prostate cancer epidemiology, Lancet 361 (2003), 955-964.

[20] C.A. Eads, K.D. Danenberg, K. Kawakami, L.B. Saltz, C. Blake, D. Shibata, P.V. Danenberg and P.W. Laird, MethyLight: a high-throughput assay to measure DNA methylation, Nucleic Acids Research 28 (2000), E32.

[21] H. Enokida, H. Shiina, M. Igawa, T. Ogishima, T. Kawakami, W.W. Bassett, J.W. Anast, L.C. Li, S. Urakami, M. Terashima, M. Verma, M. Kawahara, M. Nakagawa, C.J. Kane, P.R. Carroll and R. Dahiya, CpG hypermethylation of MDR1 gene contributes to the pathogenesis and progression of human prostate cancer, Cancer Research 64 (2004), 5956-5962.

[22] H. Enokida, H. Shiina, S. Urakami, M. Igawa, T. Ogishima, L.C. Li, M. Kawahara, M. Nakagawa, C.J. Kane, P.R. Carroll and R. Dahiya, Multigene methylation analysis for detection and staging of prostate cancer, Clinical Cancer Research $\mathbf{1 1}$ (2005), 6582-6588.

[23] H. Enokida, H. Shiina, S. Urakami, M. Igawa, T. Ogishima, D. Pookot, L.C. Li, Z.L. Tabatabai, M. Kawahara, M. Nakagawa, C.J. Kane, P.R. Carroll and R. Dahiya, Ethnic grouprelated differences in CpG hypermethylation of the GSTPI gene promoter among african-american, caucasian and asian patients with prostate cancer, International Journal of Cancer 116 (2005), 174-181.

[24] H. Enokida, H. Shiina, S. Urakami, M. Terashima, T Ogishima, L.C. Li, M. Kawahara, M. Nakagawa, C.J. Kane, P.R. Carroll, M. Igawa and R. Dahiya, Smoking influences aberrant $\mathrm{CpG}$ hypermethylation of multiple genes in human prostate carcinoma, Cancer 106 (2005), 79-86.

[25] J.I. Epstein, Diagnosing adenocarcinoma of the prostate on needle biopsy, in: Prostate Biopsy Interpretation, J.I. Epstein, ed., Lippincott-Raven, New York, 1995, pp. 87-132.

[26] M. Esteller, P.G. Corn, S.B. Baylin and J.G. Herman, A gene hypermethylation profile of human cancer, Cancer Research 61 (2001), 3225-3229.

[27] C. Goessl, R. Heicappell, R. Munker, P. Anker, M. Stroun, H. Krause, M. Muller and K. Miller, Microsatellite analysis of plasma DNA from patients with clear cell renal carcinoma, Cancer Research 58 (1998), 4728-4732.

[28] C. Goess1, H. Krause, M. Muller, R. Heicappell, M. Schrader, J. Sachsinger and K. Miller, Fluorescent methylation-specific polymerase chain reaction for DNA-based detection of prostate cancer in bodily fluids, Cancer Research 60 (2000), 5941-5945.

[29] C. Goessl, M. Muller, R. Heicappell, H. Krause, B. Straub, M. Schrader and K. Miller, DNA-based detection of prostate cancer in urine after prostatic massage, Urology 58 (2001), 335-338.

[30] C. Goessl, M. Muller, R. Heicappell, H. Krause and K. Miller, DNA-based detection of prostate cancer in blood, urine, and ejaculates, Annals of the New York Academy of Sciences 945 (2001), 51-58.

[31] M.L. Gonzalgo, C.P. Pavlovich, S.M. Lee and W.G. Nelson, Prostate cancer detection by GSTP1 methylation analysis of postbiopsy urine specimens, Clinical Cancer Research 9 (2003), 2673-2677.

[32] W.M. Grady, A. Rajput, J.D. Lutterbaugh and S.D. Markowitz, Detection of aberrantly methylated hMLH1 promoter DNA in the serum of patients with microsatellite unstable colon cancer, Cancer Research 61 (2001), 900-902.

[33] H. Gronberg, Prostate cancer epidemiology, Lancet 361 (2003), 859-864.

[34] J.A. Hanson, J.W. Gillespie, A. Grover, M.A. Tangrea, R.F. Chuaqui, M.R. Emmert-Buck, J.A. Tangrea, S.K. Libutti, W.M. Linehan and K.G. Woodson, Gene promoter methylation in prostate tumor-associated stromal cells, Journal of the National Cancer Institute 98 (2006), 255-261.

[35] S.V. Harden, H. Sanderson, S.N. Goodman, A.A. Partin, P.C. Walsh, J.I. Epstein and D. Sidransky, Quantitative GSTP1 methylation and the detection of prostate adenocarcinoma in sextant biopsies, Journal of the National Cancer Institute $\mathbf{9 5}$ (2003), 1634-1637.

[36] J.D. Hayes and D.J. Pulford, The glutathione S-transferase supergene family: regulation of GST and the contribution of the isoenzymes to cancer chemoprotection and drug resistance, Critical Reviews in Biochemistry and Molecular Biology 30 (1995), 445-600.

[37] C.A. Heid, J. Stevens, K.J. Livak and P.M. Williams, Real time quantitative PCR, Genome Research 6 (1996), 986-994.

[38] R. Henrique, C. Jerónimo, M.O. Hoque, S. Nomoto, A.L. Carvalho, V.L. Costa, J. Oliveira, M.R. Teixeira, C. Lopes and D. Sidransky, MTIG hypermethylation is associated with higher tumor stage in prostate cancer, Cancer Epidemiology Biomarkers and Prevention 14 (2005), 1274-1278.

[39] R. Henrique, C. Jerónimo, M.R. Teixeira, M.O. Hoque, A.L. Carvalho, I. Pais, F.R. Ribeiro, J. Oliveira, C. Lopes and D. Sidransky, Epigenetic heterogeneity of high-grade prostatic intraepithelial neoplasia: clues for clonal progression in prostate carcinogenesis, Molecular Cancer Research 4 (2006), 1-8.

[40] J.G. Herman, J.R. Graff, S. Myohanen, B.D. Nelkin and S.B. Baylin, Methylation-specific PCR: a novel PCR assay for methylation status of $\mathrm{CpG}$ islands, Proceedings of the National Academy of Sciences USA 93 (1996), 9821-9826.

[41] R.M. Hoffman, F.D. Gilliland, J.W. Eley, L.C. Harlan, R.A. Stephenson, J.L. Stanford, P.C. Albertson, A.S. Hamilton, W.C. Hunt and A.L. Potosky, Racial and ethnic differences in advanced-stage prostate cancer: the Prostate Cancer Outcomes Study, Journal of the National Cancer Institute 93 (2001), 388-395.

[42] M.O. Hoque, O. Topaloglu, S. Begum, R. Henrique, E. Rosenbaum, W. Van Criekinge, W.H. Westra and D. Sidransky, Quantitative methylation-specific polymerase chain reaction gene patterns in urine sediment distinguish prostate cancer patients from control subjects, Journal of Clinical Oncology 23 (2005), 6569-6575.

[43] C. Hughes, A. Murphy, C. Martin, O. Sheils and J. O'Leary, Molecular pathology of prostate cancer, Journal of Clinical Pathology 58 (2005), 673-684.

[44] W.B. Isaacs ad J.T. Isaacs, Molecular genetics of prostate cancer progression, in: Principles and Practice of Genitouri- 
nary Oncology, D. Raghavan, H.I. Scher, S.A. Leibel and P.H Lange, eds, Lippincott-Raven, Philadelphia, 1996, pp. 403408.

[45] J.P. Issa, Y.1. Ottaviano, P. Celano, S.R. Hamilton, N.E. Davidson and S.B. Baylin, Methylation of the oestrogen receptor $\mathrm{CpG}$ island links ageing and neoplasia in human colon, Nature Genetics 7 (1994), 536-540.

[46] A.B. Jani and S. Hellman, Early prostate cancer: clinical decision-making, Lancet 361 (2003), 1045-1053.

[47] A. Jemal, T. Murray, E. Ward, A. Samuels, R.C. Tiwari, A. Ghafoor, E.J. Feuer and M.J. Thun, Cancer statistics, 2005, CA: A Cancer Journal for Clinicians 55 (2005), 10-30.

[48] C. Jerónimo, H. Usadel, R. Henrique, J. Oliveira, C. Lopes, W.G. Nelson and D. Sidransky, Quantitation of GSTP1 methylation in non-neoplastic prostatic tissue and organ-confined prostate adenocarcinoma, Journal of the National Cancer Institute 93 (2001), 1747-1752.

[49] C. Jerónimo, G. Varzim, R. Henrique, J. Oliveira, M.J. Bento, C. Silva, C. Lopes and D. Sidransky, I105V polymorphism and promoter methylation of the GSTP1 gene in prostate adenocarcinoma, Cancer Epidemiology Biomarkers and Prevention 11 (2002), 445-450.

[50] C. Jerónimo, H. Usadel, R. Henrique, C. Silva, J. Oliveira, C. Lopes and D. Sidransky, Quantitative GSTP1 hypermethylation in bodily fluids of patients with prostate cancer, Urology 60 (2002), 1131-1135.

[51] C. Jerónimo, R. Henrique and D. Sidransky, Uses of DNA methylation in cancer diagnosis and risk assessment, in: DNA methylation. Approaches, Methods and Applications, M. Esteller, ed., CRC Press, Boca Raton, 2004, pp. 11-26.

[52] C. Jerónimo, R. Henrique, M.O. Hoque, F.R. Ribeiro, J. Oliveira, D. Fonseca, M.R. Teixeira, C. Lopes and D. Sidransky, Quantitative $R A R \beta 2$ hypermethylation: a promising prostate cancer marker, Clinical Cancer Research 10 (2004), 4010-4014.

[53] C. Jerónimo, R. Henrique, M.O. Hoque, E. Mambo, F.R. Ribeiro, G. Varzim, J. Oliveira, M.R. Teixeira, C. Lopes and D. Sidransky, A quantitative methylation profile of prostate cancer, Clinical Cancer Research 10 (2004), 8472-8478.

[54] G.H. Kang, S. Lee, H.J. Lee and K.S. Hwang, Aberrant CpG island hypermethylation of multiple genes in prostate cancer and prostatic intraepithelial neoplasia, Journal of Pathology 202 (2004), 233-240.

[55] D.W. Keetch, W.J. Catalona and D.S. Smith, Serial prostatic biopsies in men with persistently elevated serum prostate specific antigen values, Journal of Urology 151 (1994), 15711574.

[56] N. Konishi, M. Nakamura, M. Kishi, M. Nishimine, E. Ishida and K. Shimada, Heterogeneous methylation and deletion patterns of the INK4a/ARF locus within prostate carcinomas, American Journal of Pathology 160 (2002), 1207-1214.

[57] J.S. Lam, Y.K. Cheung, M.C. Benson and E.T. Goluboff, Comparison of the predictive accuracy of serum prostate specific antigen levels and prostate specific antigen density in the detection of prostate cancer in Hispanic-American and white men, Journal of Urology 170 (2003), 451-456.

[58] W.H. Lee, R.A. Morton, J.I. Epstein, J.D. Brooks, P.A. Campbell, G.S. Bova, W.S. Hsieh, W.B. Isaacs and W.G. Nelson, Cytidine methylation of regulatory sequences near the pi-class glutathione S-transferase gene accompanies human prostatic carcinogenesis, Proceedings of the National Academy of Sciences USA 91 (1994), 11733-11737.

[59] W.H. Lee, W.B. Isaacs, G.S. Bova and W.G. Nelson, CG island methylation changes near the GSTP1 gene in prostatic carcinoma cells detected using the polymerase chain reaction: a new prostate cancer biomarker, Cancer Epidemiology Biomarkers and Prevention 6 (1997), 443-450.

[60] L.C. Li, H. Zhao, K. Nakajima, B.R. Oh, L.A. Filho, P. Carroll and R. Dahiya, Methylation of the E-cadherin gene promoter correlates with progression of prostate cancer, Journal of Urology 166 (2001), 705-709.

[61] L.C. Li, S.T. Okino and R. Dahiya, DNA methylation in prostate cancer, Biochimica et Biophysica Acta 1704 (2004), 87-102.

[62] L.C. Li, H. Shiina, M. Deguchi, H. Zhao, S.T. Okino, C.J. Kane, P.R. Carroll, M. Igawa and R. Dahiya, Age-dependent methylation of ESR 1 gene in prostate cancer, Biochemical and Biophysical Research Communications 321 (2004), 455-461.

[63] L.C. Li, P.R. Carroll and R. Dahiya, Epigenetic changes in prostate cancer: implication for diagnosis and treatment, Journal of the National Cancer Institute 97 (2005), 103-115.

[64] X. Lin, M. Tascilar, W.H. Lee, W.J. Vles, B.H. Lee, R. Veeraswamy, K. Asgari, D. Freije, B. van Rees, W.R. Gage, G.S. Bova, W.B. Isaacs, J.D. Brooks, T.L. DeWeese, A.M. DeMarzo and W.G. Nelson, GSTP1 CpG island hypermethylation is responsible for the absence of GSTP1 expression in human prostate cancer cells, American Journal of Pathology 159 (2001), 1815-1826.

[65] C.A. Macintosh, M. Stower, N. Reid and N.J. Maitland, Precise microdissection of human prostate cancers reveals genotypic heterogeneity, Cancer Research 58 (1998), 23-28.

[66] P. Marks, R.A. Rifkind, V.M. Richon, R. Breslow, T. Miller and W.K. Kelly, Histone deacetylases and cancer: causes and therapies, Nature Reviews in Cancer 1 (2001), 194-202.

[67] R. Maruyama, S. Toyooka, K.O. Toyooka, A.K. Virmani, S. Zochbauer-Muller, A.J. Farinas, J.D. Minna, J. McConnell, E.P. Frenkel and A.F. Gazdar, Aberrant promoter methylation profile of prostate cancers and its relationship to clinicopathological features, Clinical Cancer Research 8 (2002), 514-519.

[68] R.B. Nadler, S. Loeb, K.A. Roehl, J.A. Antenor, S. Eggener and W.J. Catalona, Use of $2.6 \mathrm{ng} / \mathrm{ml}$ prostate specific antigen prompt for biopsy in men older than 60 years, Journal of Urology 174 (2005), 2154-2157.

[69] M. Nakayama, M.L. Gonzalgo, S. Yegnasubramanian, X. Lin, A.M. DeMarzo and W.G. Nelson, GSTP1 CpG island hypermethylation as a molecular biomarker for prostate cancer, Journal of Cell Biochemistry 91(2004), 540-552.

[70] D.E. Neal and J.L. Donovan, Prostate cancer: to screen or not to screen? Lancet Oncology 1 (2000), 17-24.

[71] W.G. Nelson, A.M. DeMarzo and T.L. DeWeese, The molecular pathogenesis of prostate cancer: implications for prostate cancer prevention, Urology 57 (2001), 39-45.

[72] W.G. Nelson, A.M. DeMarzo and W.B. Isaacs, Prostate cancer, New England Journal of Medicine 349 (2003), 366-381.

[73] M. Noguchi, T.A. Stamey, J.E. McNeal and R. Nolley, Prognostic factors for multifocal prostate cancer in radical prostatectomy specimens: lack of significance of secondary cancers, Journal of Urology 170 (2003), 459-463.

[74] T. Ogishima, H. Shiina, J.E. Breault, L. Tabatabai, W.W. Bassett, H. Enokida, L.C. Li, T. Kawakami, S. Urakami, L.A. Ribeiro-Filho, M. Terashima, M. Fujime, M. Igawa and R. Dahiya, Increased heparanase expression is caused by promoter hypomethylation and up-regulation of transcriptional factor early growth response-1 in human prostate cancer, Clinical Cancer Research 11 (2005), 1028-1036.

[75] A. Olek, J. Oswald and J. Walter, A modified and improved method for bisulphite based cytosine methylation analysis, Nucleic Acids Research 24 (1996), 5064-5066. 
[76] P. Pakneshan, M. Szyf and S.A. Rabbani. Hypomethylation of urokinase (uPA) promoter in breast and prostate cancer: prognostic and therapeutic implications, Current Cancer Drug Targets 5 (2005), 471-488.

[77] P. Pakneshan, R.H. Xing and S.A. Rabbani, Methylation status of UPA promoter as a molecular mechanism regulating prostate cancer invasion and growth in vitro and in vivo, FASEB Journal 17 (2003), 1081-1088.

[78] K.A. Rauen, D. Sudilovsky, J.L. Le, K.L. Chew, B. Hann, V. Weinberg, L.D. Schmitt and F. McCormick, Expression of the coxsackie adenovirus receptor in normal prostate and in primary and metastatic prostate carcinoma: potential relevance to gene therapy, Cancer Research 62 (2002), 3812-3818.

[79] E. Rosenbaum, M.O. Hoque, Y Cohen, M. Zahurak, M.A. Eisenberger, J.I. Epstein, A.W. Partin and D. Sidransky, Promoter hypermethylation as an independent prognostic factor for relapse in patients with prostate cancer following radical prostatectomy, Clinical Cancer Research 11 (2005), 83218325.

[80] E.T. Ruijter, G.J. Miller, C.A. van de Kaa, A. van Bokhoven, M.J. Bussemakers, F.M. Debruyne, D.J. Ruiter and J.A Schalken, Molecular analysis of multifocal prostate cancer lesions, Journal of Pathology 188 (1999), 271-277.

[81] F.H. Schroder, I. van der Cruijsen-Koeter, H.J. de Koning, A.N. Vis, R.F. Hoedemaeker and R. Kranse, Prostate cancer detection at low prostate specific antigen, Journal of Urology 163 (2000), 806-812.

[82] W.A. Schulz, J.P. Elo, A.R. Florl, S. Pennanen, S. Santourlidis, R. Engers, M. Buchardt, H.H. Seifert and T. Visakorpi, Genomewide DNA hypomethylation is associated with alterations on chromosome 8 in prostate carcinoma, Genes Chromosomes Cancer 35 (2002), 58-65.

[83] D.B. Seligson, S. Horvath, T. Shi, H. Yu, S. Tze, M. Grunstein and S.K. Kurdistani, Global histone modification patterns predict risk of prostate cancer recurrence, Nature 435 (2005), 1262-1266.

[84] R. Singal, L. Ferdinand, I.M. Reis and J.J. Schlesselman, Methylation of multiple genes in prostate cancer and the relationship with clinicopathological features of disease, Oncology Reports 12 (2004), 631-637.

[85] M.H. Sokoloff, X.J. Yang, M. Fumo, D. Mhoon and C.B. Brendler, Characterizing prostatic adenocarcinomas in men with a serum prostate specific antigen level of $<4.0 \mathrm{ng} / \mathrm{mL}$, British Journal of Urology International 93 (2004), 499-502.

[86] D.M. Steinberg, J. Sauvageot, S. Piantadosi and J.I. Epstein, Correlation of prostate needle biopsy and radical prostatectomy Gleason grade in academic and community settings, American Journal of Surgical Pathology 21 (1997), 566-576.
[87] C.I. Suh, T. Shanafelt, D.J. May, K.R. Shroyer, J.B. Bobak, E.D. Crawford, G.J. Miller, N. Markham and L.M. Glode, Comparison of telomerase activity and GSTP1 promoter methylation in ejaculate as potential screening tests for prostate cancer, Molecular Cell Probes 14 (2000), 211-217.

[88] I.M. Thompson, P.J. Goodman, C.M. Tangen, M.S. Lucia, G.J. Miller, L.G. Ford, M.M. Lieber, R.D. Cespedes, J.N. Atkins, S.M. Lippman, S.M. Carlin, A. Ryan, C.M. Szczepanek, J.J. Crowley and C.A. Coltman Jr., The influence of finasteride on the development of prostate cancer, New England Journal of Medicine 349 (2003), 215-224.

[89] T. Tokizane, H. Shiina, M. Igawa, H. Enokida, S. Urakami, T. Kawakami, T. Ogishima, S.T. Okino, L.C. Li, Y. Tanaka, N. Nonomura, A. Okuyama and R. Dahiya, Cytochrome P450 1B1 is overexpressed and regulated by hypomethylation in prostate cancer, Clinical Cancer Research 11 (2005), 5793 5801.

[90] Y. Tokumaru, S.V. Harden, D.I. Sun, K. Yamashita, J.I. Epstein and D. Sidransky, Optimal use of a panel of methylation markers with GSTP1 hypermethylation in the diagnosis of prostate adenocarcinoma, Clinical Cancer Research $\mathbf{1 0}$ (2004), 5518-5522.

[91] D.A. Troyer, J. Mubiru, R.J. Leach and S.L. Naylor, Promise and challenge: markers of prostate cancer detection, diagnosis and prognosis, Disease Markers 20 (2004), 117-128.

[92] D.J. Weisenberger, M. Campan, T.I. Long, M. Kim, C. Woods, E. Fiala, M. Ehrlich and P.W. Laird. Analysis of repetitive element DNA methylation by MethyLight, Nucleic Acids Research 33 (2005), 6823-6836.

[93] K. Woodson, R. Hayes, L. Wideroff, L. Villaruz and J. Tangrea, Hypermethylation of GSTP1, CD44, and E-cadherin genes in prostate cancer among US Blacks and Whites, Prostate 55 (2003), 199-205.

[94] M. Yamanaka, M. Watanabe, Y. Yamada, A. Takagi, T. Murata, H. Takahashi, H. Suzuki, H. Ito, H. Tsukino, T. Katoh, Y. Sugimura and T. Shiraishi, Altered methylation of multiple genes in carcinogenesis of the prostate, International Journal Cancer 106 (2003), 382-387.

[95] Y. Yegnasubramanian, J. Kowalski, M.L. Gonzalgo, M. Zahurak, S. Piantadosi, P.C. Walsh, G.S. Bova, A.M. DeMarzo, W.B. Isaacs and W.G. Nelson, Hypermethylation of CpG islands in primary and metastatic human prostate cancer, Cancer Research 64 (2004), 1975-1986.

[96] M. Zhou, Y. Tokumaru, D. Sidransky and J.I. Epstein, Quantitative GSTP1 methylation levels correlate with Gleason grade and tumor volume in prostate needle biopsies, Journal of Urology 171 (2004), 2195-2198. 


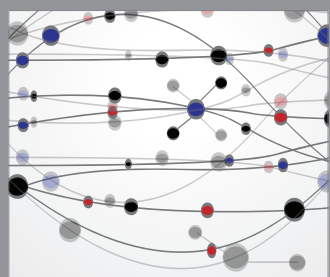

The Scientific World Journal
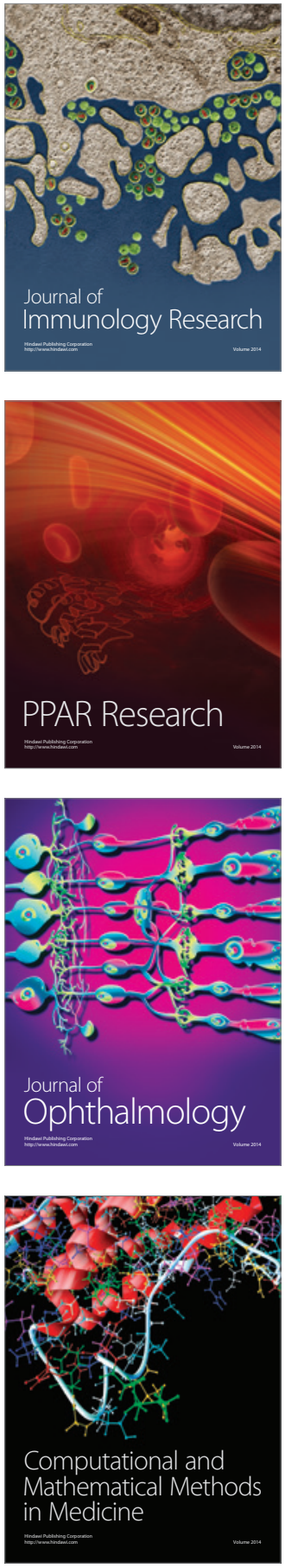

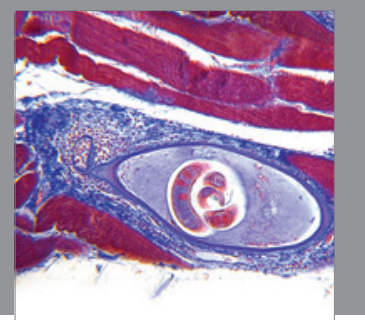

Gastroenterology

Research and Practice
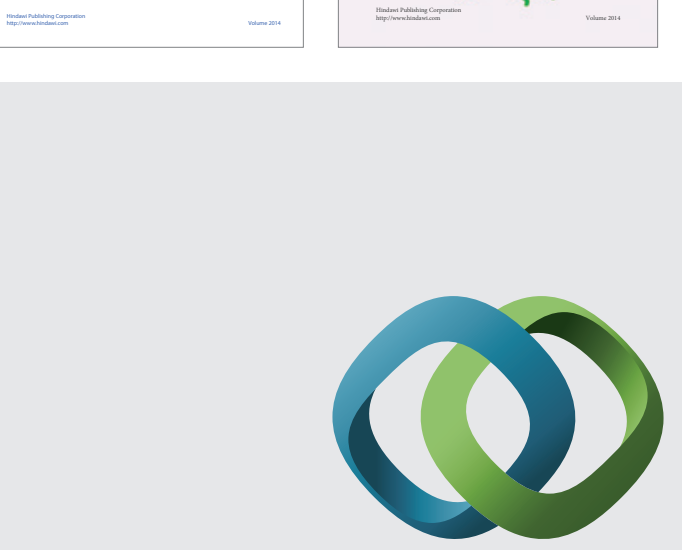

\section{Hindawi}

Submit your manuscripts at

http://www.hindawi.com
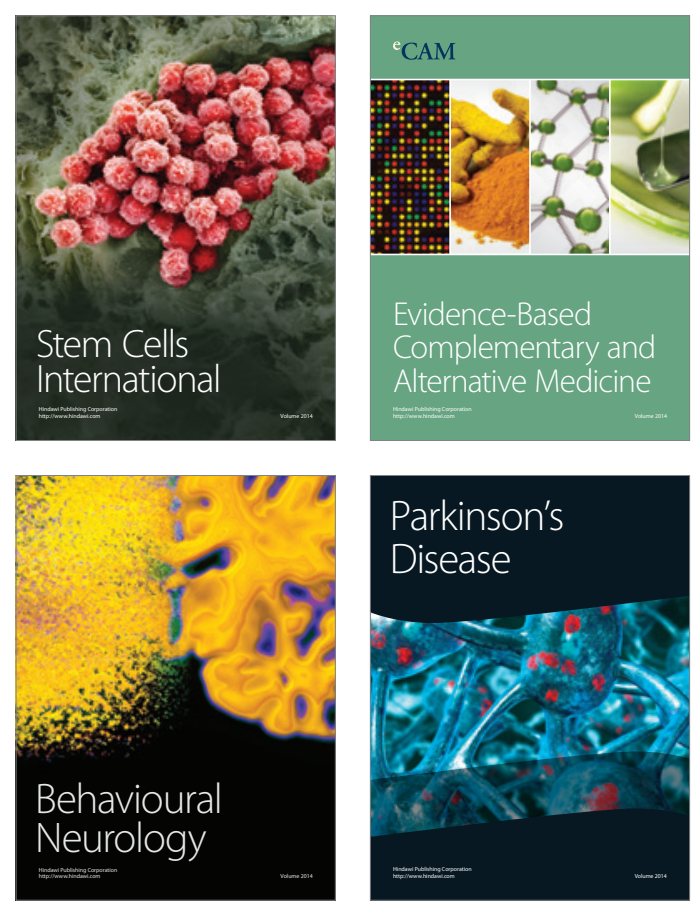

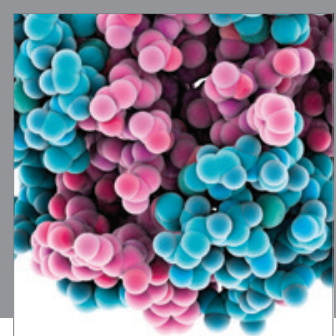

Journal of
Diabetes Research

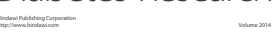

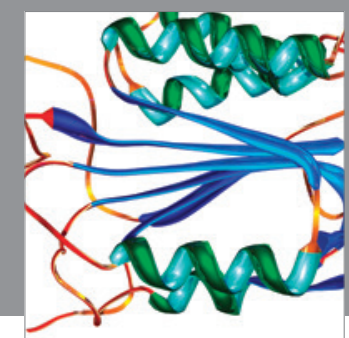

Disease Markers
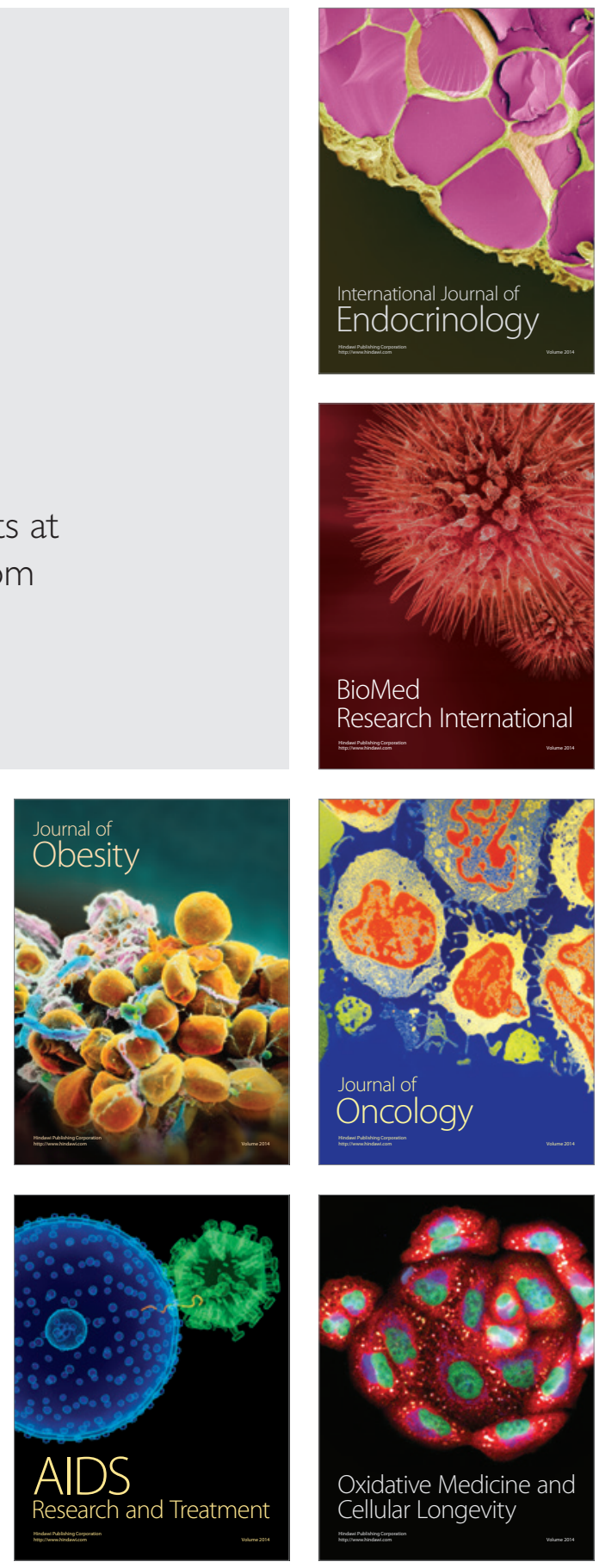